\title{
Virtual Power System: Novel approach for Distributed Generation and Consumption Coordination
}

\author{
E. Cagno ${ }^{1}$, F. Castelli Dezza ${ }^{2}$, M. Delfanti ${ }^{3}$, M. Merlo ${ }^{3}$, and A. Trianni ${ }^{1}$ \\ ${ }^{1}$ Department of Management, Economics and Industrial Engineering \\ 2 Department of Mechanical Engineering \\ ${ }^{3}$ Energy Department \\ Politecnico di Milano \\ Via Lambruschini, 20156 - Milano - Italy \\ e-mail: enrico.cagno@polimi.it; francesco.castellidezza@polimi.it; maurizio.delfanti@polimi.it; marco.merlo@polimi.it; \\ andrea.trianni@polimi.it
}

\begin{abstract}
Worldwide the introduction of dispersed generators (DG) in the distribution network is assuming a significant importance. There is an increasing relevance of the energy process efficiency improvement; as for electric power systems, the most interesting perspective concerns the capability of the system to increase the exploitation of the renewable resources. The integration of DGs in the electric distribution network requires a revision of this infrastructure, so far designed and developed assuming that power flows in one direction: from the high voltage transmission network to the medium voltage distribution, to reach final customers on the low voltage network.

The attention to an efficient operation of distribution networks is increasing all over the world; this interest is becoming higher and higher also in Italy, where the high energy prices push in the direction of fostering efficiency as much as possible.

This work describes a study developed in the Alpenergy project framework: an International Cooperation Program aimed at introducing an efficient operational model for the distributed production and consumption. In particular it is proposed a new model for the integration and the management of the DG in the distribution network. The new model (defined VPS: Virtual Power System) is based on a communication channel between the active users (generators), the loads and, eventually, the Distribution System Operators (DSOs).
\end{abstract}

\section{Key words}

renewable energy sources, distributed generation, distributed consumption, virtual power plant.

\section{Introduction}

The global concern on energy is rapidly growing mainly due to the increase of demand of primary energy, especially in developing countries, which is estimated to be about $1.7 \%$ per year between 2004 and 2030 [1]. But, the increase of primary energy is, at the moment, accompanied by high levels of GHG-emissions: the performance of many developed countries that signed the Kyoto Protocol are quite far from what required, and it is estimated that developing countries will account for over three-quarters of the increase of global $\mathrm{CO} 2$ emissions between 2004 and 2030 [2]. China alone is responsible for $39 \%$ of the rise in global emissions, due to its carbonintensive economics and will overtake the United States as the world's biggest emitter before 2010 [3-4].

Within this scenario implementing measures able to reduce the consumption - thus increasing the energy efficiency - especially in the industrial sector, that covers alone more than one-half of the world's total delivered energy [1], is surely quite useful, but it does not seem to be the unique solution. It seems more profitable to respond to the world's energy needs pursuing also different paths: one is represented by enhancing the share on the total of Renewable Energy Sources (then RES), quite promising but at the moment not economically competitive, with the exception of hydroelectric power, with conventional fossil fuelled-generation except from territories whereas investments in improving the grid due to the connection of large centralized power plants to the net - would result to be too expensive. For this reason it is widely accepted that RES deployment would be driven only with strong Government policies and incentives.

Furthermore, some other peculiarities of non-hydro RES are considered as a physical barrier to their deployment in high-density territories:

-low energy density, compared to conventional technologies, thus requiring a larger space for the same power installed in a site;

-dependence on climate conditions: at the moment RES with a large potential, like solar and wind, provide energy in an intermittent manner, whilst biomasses and biogas are able to provide electricity on a $24 \mathrm{~h}$ basis.

But there is also a cultural barrier: this common knowledge is due to the fact that, no matter of highdensity or low-density territories, the system does not, at the moment, integrate production and consumption. The 
electrical system has always been thought just according to the needs of consumers: for this reason, the more power needed, the more installed and delivered (with the consequent improvement, where necessary, of the grid).

This is true also for RES, and proven by the fact that, even for $100 \%$ green supply consumers, at the moment it is guaranteed only that the overall energy consumed (and bought) is equal to the energy that the system has produced (maybe somewhere else) over a long period, e.g. one year: this mismatch between production and consumption would result in just an economical balance between energy inputs and outputs.

If the aim is to have an effective energy sustainability within a territory, it should be necessary to re-think the overall electrical system in order to take into account not only the needs of consumers, but also the constraints of producers (since, for example, RES within a territory are limited) and the limitations of the distribution network (since the energy that can be delivered is limited). In an effective sustainable energy scenario, the energy consumed in a territory should be entirely produced in the same territory, and better if produced from RES.

To do that, it seems clear that a holistic approach, able to involve producers, distributors, consumers and decisionmakers is needed.

If this could be someway far from the application in high density territories, it seems viable in many regions of the Alpine Space, characterized by:

-scattered habitats (low density of population and low consumption),

-large availability of space and

-high costs/environmental difficulties in the expansion of the existing grid infrastructures grid.

In those regions grid operators (distributors) face quite often the reluctance of local communities when building new lines in order to reach remote customers and thus may look favorably to a real integration of RES with consumption.

\section{THE ALPENERGY PROJECT}

\section{A. Who's in?}

Developed within the Interreg IIIB of the Alpine Space and jointly financed by the European Union, through the European Regional Development Fund (ERDF) and the Partner States (Member and non-Members) taking part in the activities, the AlpEnergy project is an International Cooperation Program aiming at introducing an efficient operational model for the distributed production and consumption with RES.

Since, as expressed before, the issue of energy in the Alpine Space cannot be faced just considering producers or consumers separately, AlpEnergy brings together power suppliers, development agencies, public regional policy makers, research institutes and government administrations from different countries of the Alpine space (France, Switzerland, Italy, Germany, Slovenia) $[5]$.

\section{B. Project's objectives}

There are several topics that need to be studied in order to reach the main objective, that is to create and test an integrated system able to match instantaneously distributed generation system (powered by RES) and distributed consumption that will be valid everywhere, providing pilot applications within portions of the Alpine Space region. The objectives of the project could be summarized as follows [6]:

-To capitalize existing potentials for electricity production from endogenous renewable resources and for demand management in existing economic sectors.

-To provide new-knowledge based income and business opportunities for farmers and traditional and innovative enterprises.

-To contribute towards security of affordable electricity supply in the Alpine Space, thus supporting the competitiveness of Alpine enterprises in general.

-To provide a basis for new electricity supply concepts reducing the number of electricity grid lines in, and impact on, the landscape and for cleaner electricity generation, thus reducing the global and local environmental impact of electricity generation.

-To enhance worldwide business opportunities for Alpine enterprises and to contribute to growth, employment and sustainable development by making the Alpine Space a showcase for sustainable electricity supply with a strong vibrancy towards other mountain areas in the world.

To reach these objectives, it is necessary to define and describe the basic element of the project, the integrated model for distributed production and consumption with RES, called Virtual Power System (then VPS), in order to evaluate its characteristics and possible applications. Within this project the Italian team has devoted its attention to the definition of a version of the VPS, valid everywhere but specifically fitting the characteristics of the Italian electrical system and context.

\section{DISPERSED GENERATION: THE NEW SCENARIO}

Nowadays, the technology evolution made available very interesting solutions for the exploitation of the renewable energy (solar energy, biogas, biomass, etc.), or for a more efficient energy conversion (microturbines, highefficiency cogeneration plants, etc), with "domestic" rated powers. This evolution made it possible to spread a series of small dispersed generators, capable of fully exploiting the resource locally available.

The integration of DG in the electric distribution network requires a revision of this infrastructure and of its protection and control devices, so far designed and developed assuming that power flows in one direction (from the transmission/subtransmission network to the distribution network) to reach final customers connected to the low voltage (LV) network, as expressed in the introduction. In fact the presence of DG on the distribution network may cause an Inverse Power Flow (IPF), i.e. a flow from a medium voltage (MV) network to the high voltage (HV) network.

The growing penetration of Dispersed Generation (DG) in Medium Voltage (MV) and Low Voltage (LV) grids is 
an issue to be faced by Distribution System Operators (DSOs), as MV and LV grids have been designed and operated so far with the objective to feed customers and not to connect generators.

Several operational problems may arise when too many generators, even small ones, are connected under the same high voltage / medium voltage (HV/MV) transformer. These problems are related to:

-current flows across branches,

-Supply Voltage Variations (SVV), i.e. supply voltage variations for each bus,

-Rapid Voltage Changes (RVC), associated to sudden variations of generator power output,

-short circuit currents,

-protection systems,

-direction of power flows,

-grid losses.

The problems cover a wide area in the Power System regulation and control [7, 8, 9], resulting to be very complex and motivating a new model for the network integration and control of the DG.

The new integrated model has been defined Virtual Power System (VPS); in the following section the definition and description of this new paradigm has been detailed.

\section{DISPERSED GENERATION: THE NEW SCENARIO}

Virtual Power Systems (VPS) are systems of distributed power production and distributed consumption, and a distribution network, along with a proper communication network, characterized by being based on Renewable Energy Sources; production and consumption are balanced both on energy and power.

The spatial extension of those systems may differ from country to country. Respect to the electric network, the model has to cover different areas, ranging from production, to transmission, distribution and consumption; in the following a short description is introduced for each one of these topics.

\section{Description of production:}

The power production is supposed to be provided using one or more RES technologies and by one or more power plants, in order to shape a single energy production profile. Considering only the production, VPS have some similarities to Virtual Power Plants (then VPPs).

\section{Description of consumption:}

As for energy production, one or more loads can be aggregated to shape a single energy consumption profile (VPP).

Exploiting a proper communication network, VPS are able to balance as far as possible the two (generation and loads) energy profiles.

In case of mismatch (e.g. very small applications) an energy buffer could be needed prior to accessing to the main network.

\section{Description of distribution:}

The power distribution can be performed using either the existing network or an "ad hoc" network (i.e. for scattered habitats with very remote customers).

In the former case, the existing network can serve also as an energy buffer and/or as a back-up system.

In the case of network as a back-up system, the electrical service continuity surely increases-

The use of the network as an energy buffer is a possibility but it could not be compliant with respect to the overall efficiency if the penetration of renewable (not dispatchable or controllable) generation reach high values.

On the other hand, to fully exploit the concept of VPS, consumers should shape their profile according to the production plant technology, potentials and management. In the latter case, the "ad hoc" network can increase the service continuity but back-up cannot be guaranteed.

To this extent, a VPS represents a valid solution for areas with difficulties in accessing the main network, or where investments in improving the network would be not economically justifiable for both stakeholders and the community.

The decrease of communication costs and the larger availability of new technologies made effective to implement all the VPS potentials.

VPS should be evaluated considering a holistic approach of the project's sustainability: environmental impact, overall costs, service continuity are variables to measure the efficiency and efficacy of RES power systems.

\section{APPROACH PROPOSED}

In the Alpenergy framework the VPS deployment has been approached with respect to three different steps:

-The first step regards the definition of the loads and generators to be included into the VSP. For each one the total energy requirement (on a year timescale) and the daily load curve (subdivided with respect to the season variation and considering holiday and working days) have been defined. In particular, the load curve (load variation for each considered day; LC in the following) has been defined with a 4 sample/hour precision (i.e. one sample for each quarter of hour). In this step all the available information about the network in analysis, the load energy requirements and the relative LC has been collected. The step 1 can be identified as a preliminary study, aimed at evaluating the theoretical improvement, in the distribution networks, achievable thanks to the VPS approach. Unfortunately, at this stage of the research, a complete database containing energy requirements and the LCs for many loads is not yet available, thus, data from the most recent literature will be assumed [11].

-The second step is based on the evaluation of the results obtained by step 1 in order to identify the loads and the generators that should be included in the VPS to maximize its performance. For these loads and generators 
a dedicated monitoring infrastructure has to be installed, aiming at collecting the real data (energy requirements, LCs, etc.) on the power flows on one year time window. The goal of phase 2 is to complete a reliable quantification of the VPS approach capability for the area into analysis, and to complete a business plan for the economic evaluation of the project.

-Finally, the third step is dedicated to the physical deployment of the VPS.

This paper focuses on the VPS concept introduction and to some preliminary result of a Step 1 phase, applied on a real test bed in Italy.

\section{FIRST TESTS AND RESULTS}

In this paper is presented a preliminary study based on a virtual application, i.e. the Politecnico di Milano campus, integrated in a VPS.

The university has a dedicated metering system [10] that collects information about the energy consumption of each building for each quarter of hour.

The theoretical VPS analyzed includes one building of the campus in Milan and other customers: an office building, and electric lighting load, and a little swimming pool. For this loads (fictitious) the energy consumption and the LC data have been obtained adopting a literature model [11] based on: surface of the office (figure 2), number of electric lights, etc., in order to obtain a parametric approach from the literature data up to the test into the analysis.

An algorithm has been developed to collect all the uncontrollable loads and build an aggregate Virtual Load Plant (VLP) (figure 3).

Renewable generators have been introduced also, and in particular: a $30 \mathrm{~kW}$ photovoltaic application and a 279 $\mathrm{kW}$ Biogas power plant. Considering these generators, available literature data have been assumed, in terms of energy production and generation profile.

The algorithm developed considers the photovoltaic as un-controllable generation, so the only option available is to chronologically reduce the loads (figure 4).

Finally, the control law of the controllable generator (biogas) is aimed to the peak shaving of the "total consumption" of the VPS. In particular the approach takes into account the Biogas storage capability of the power plant considered, the maximum power of the electric generators installed in the Biogas power plant and other technical bounds.

It has to be pointed out how, in this preliminary approach, the only control introduced is on the Biogas Power Plant.

Figure 5 depicts an example about the result obtained, in particular the picture shows the VPS LC for a winter week, before (blue) and after (green) the control. Figure 6 is relevant to the result on a year time windows.

The theoretical VPS simulated provides very interesting results, demonstrating the huge capability of the approach in terms of relief of the power flows on the electric network.

The future work will be focused on the collection of data relevant to a real life application on the Italian system.

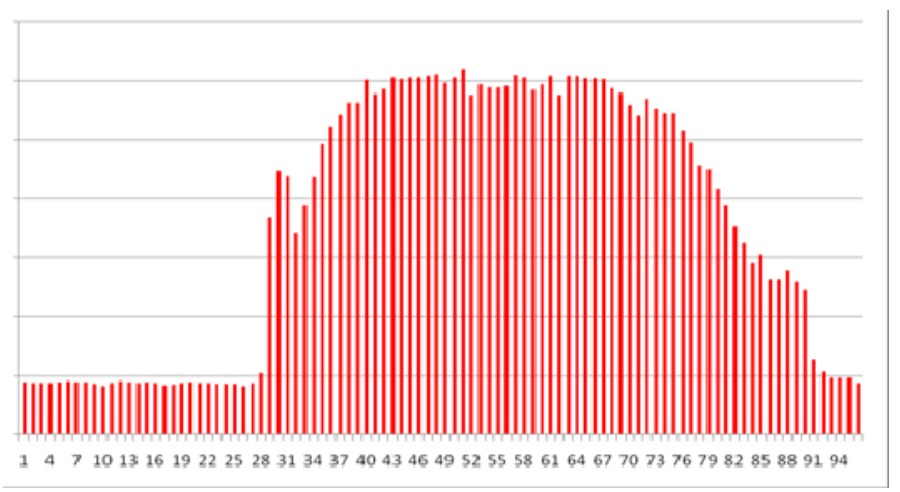

Figure 1: Measured LC for a load (school building: Politecnico Campus) for a winter working days (load vs. time - quarter of hour)

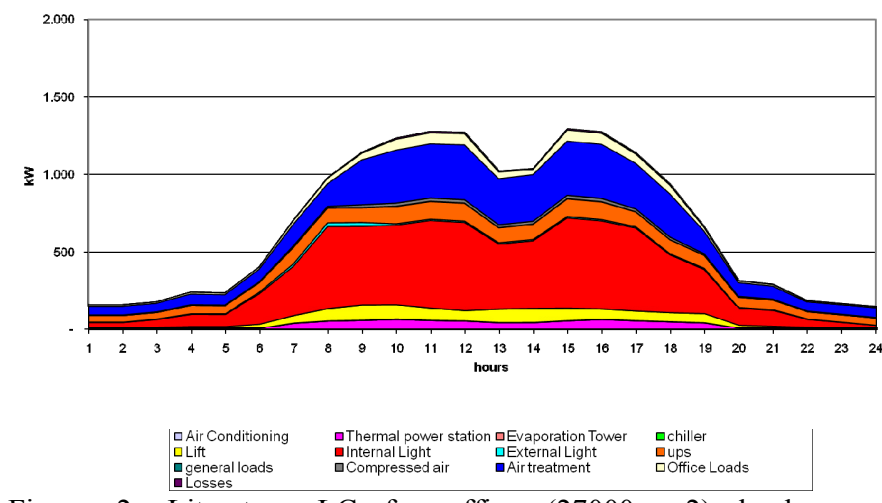

Figure 2: Literature LC for office $(27000 \mathrm{~m} 2)$ loads: subdivision for each appliance

( $\mathrm{kW}$ vs hours in a winter working day).

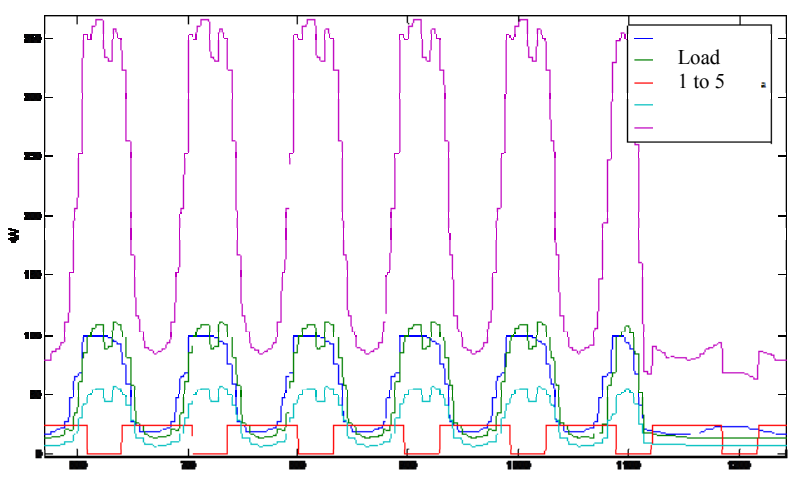

Figure 3: Elaboration of the total loads $[\mathrm{kW}] \mathrm{LC}$ in a time window of one week

(each colored line correspond to a different load)

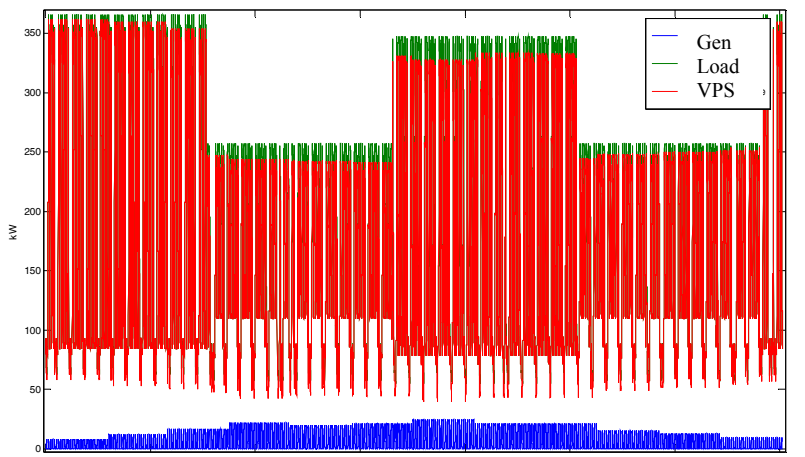

Figure 4: Elaboration of the total loads LC and renewable generators (un-controllable) in a time window of a year 


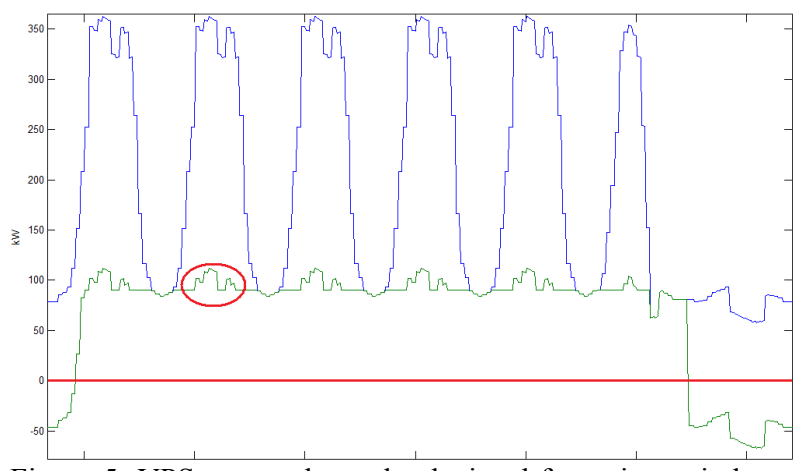

Figure 5: VPS approach results depicted for a time window of one week. (blue line refers to the total load while green line corresponds to the VPS approach proposed)

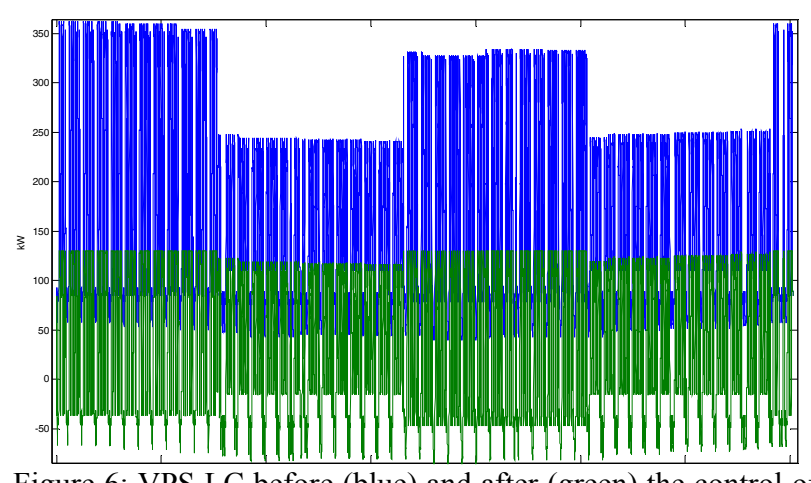

Figure 6: VPS LC before (blue) and after (green) the control of the available generators.

\section{CONCLUSION}

Respect to the increasing weight that the $D G$ is assuming in the electric network worldwide, it results more and more critical to define a new integration and regulation model that guides towards a revolution in the generation, transmission and distribution of the electric energy.

The new VPS paradigm has to be clearly defined putting in evidence its capabilities, that, if fully exploited, will make it virtuous. In this paper the attention has been focused on the Italian electrical system, introducing those characteristics considered basilar for the hypothesis of shifting from the existing regulation on the electrical system to a different regulation able to integrate, develop and boost VPS.

Local nature: considering the technical and regulatory peculiarities of VPS detailed in the previous sections, it is retained that if production and consumption would be within the same medium voltage net, thus lowering the burden on the high and very high voltage grids, there might be a reduction of losses in delivering power to the final user. What drawn above drives VPS to have a local nature.

Distributed and aggregated: Possibilities to aggregate active and passive users: the distributed nature of users within VPS is able to increase the number of users able to access to the electrical market. The aggregation of active and passive users, furthermore, would lead to have a coordinated management, from the feasibility study phase to realization and operation of the different plants, thus gaining advantages on different economies: learning by sure, but also scale and scope economies.

Synchronization: The synchrony of production and consumption seems to be essential for lowering the burden on high and very high voltage nets. Furthermore, synchronization (that can be supposed on a limited range of time, e.g. 15 minutes) is able to reduce investments and upgrading the existing network, since the overall system would result to be more efficient and particularly more efficiently managed, with a consequent reduction of back-up structures. This point is strictly related to the presence of passive users which is, somehow, a new way of thinking the overall systems: the integration, within the electrical system, of passive users enhances the opportunities to balance production and consumption, shaping the load profile according to the constraints of producers and distributors.

Some more considerations are needed in order to draw the development path towards the full implementation of the VPS concept able to exploit all its potentials:

-Business and organizational models are needed to evaluate VPSs within one region. Those models should be developed including the technical and normative constraints existing in short, medium and long terms. It seems to be important to develop business plans able to draw different scenarios according with different constraints relaxation (both due to a possible development of technical solutions and/or regulations in support of VPS). It seems important to remark that the regulatory constraints might be relaxed even farther in time compared with the technical ones, and might represents the real issue to the full deployment of VPS. Within the AlpEnergy project it is planned to develop and test a number of applications of those models within the different normative and technical contexts of each partner country.

-The transnational nature of the Alpine Space may represent a further challenge to the deployment of VPS, since a transnational approach of producing, consuming and distributing power needs a homogeneous regulation with regards to the electrical system.

-The VPS are, at the moment, defined exclusively considering RES: it seems remarkable to stress the advantages of RES towards the overall system (in terms of environmental impact and increase of share of "green power"); but VPS could be extended also outside the concept of Renewable Energy Sources, thus including high-efficiency sources of power (e.g. CHP). The resulting advantage of the overall electrical system would be surely reduced (since the share of RES would not be increased) but the 
efficiency of the system would be, in any case, enhanced.

-VPS have now be defined considering scattered habitats with several problems of service continuity and, within this, a minimum level of power quality: this solution would lead to avoid undesired investments in upgrading the existing net or building new infrastructures that would imply high costs and a high environmental impact. But the VPS concept could be extended also in a non-scattered context, thinking a VPS as a quasi-isled systems that may reduce the overall load (especially referring to peak loads, both considering power production and consumption) and thus reducing the need of adapting the grid (and related infrastructures) especially on high and very-high voltage.

\section{References}

[1] Energy Administration Agency Department of Energy, "International Energy Outlook 2009”, DOE/EIA0484(2009), Washington DC, USA.

[2] United Nations, "Kyoto Protocol Reference Manual on Accounting of Emissions and Assigned Amounts", UNFCC secretariat, New York, 2007.

[3] International Energy Agency, OECD/NEA, "World Energy Outlook 2006”, Head of Publication Office, Paris, France.

[4] F. He, D. Qin, "China's Energy Strategy in the Twenty-first Century", China \& World Economy, Vol. 14, No.2, pp. 93-104.

[5] http://www.alpenergy.net/ (visited on 6th July $2009 \mathrm{~h}$ 14:00), AlpEnergy project, Interreg IIIB.

[6] "Virtual Power System - White book", preliminary version of 23rd May 2009, AlpEnergy project, forecoming.

[7] M.H.J. Bollen, Y. Yang, F. Hassan: "Integration of Distributed Generation in the Power System - A Power Quality Approach", Proceedings Harmonics and Quality of Power, ICHQP 2008.

[8] M. Delfanti, M.S. Pasquadibisceglie, M. Pozzi, M. Gallanti, R. Vailati, "Limits to dispersed generation on Italian MV networks", Proceedings CIRED 20th Conference on Electricity Distribution, Prague, 8-11 June 2009.

[9] M. Delfanti, M. Merlo, V. Olivieri, M. Pozzi, M. Gallanti, "Power flows in the Italian distribution electric system with dispersed generation", Proceedings CIRED 20th Conference on Electricity Distribution, Prague, 8-11 June 2009.

[10] https://www.ssl.energyteam.it/

[11] http://www.ricercadisistema.it 